\title{
KNbO3 Soportado en Zeolita BETA: Evaluación del Método de Incorporación
}

\section{KNbO3 Supported onto BETA Zeolite: Evaluation of the Incorporation Method}

\section{Candelaria Leal Marchena}

Centro de Investigación y Tecnología Química (CITeQ), UTN - CONICET, Maestro Marcelo López esq. Cruz Roja Argentina, (5016) Córdoba, Argentina.

cleal@frc.utn.edu.ar

\section{Georgina Ortenzi}

Centro de Investigación y Tecnología Química (CITeQ), UTN - CONICET, Maestro Marcelo López esq. Cruz Roja Argentina, (5016) Córdoba, Argentina.

gportenzi@gmail.com

\section{Liliana Pierella}

Centro de Investigación y Tecnología Química (CITeQ), UTN - CONICET, Maestro Marcelo López esq. Cruz Roja Argentina, (5016) Córdoba, Argentina.

Ipierella@frc.utn.edu.ar

\section{Resumen}

Los óxidos mixtos presentan propiedades catalíticas; sin embargo, exhiben baja área superficial, lo que limita su uso como catalizadores. Con el fin de aumentar su superficie específica, se prepararon materiales a partir de la incorporación del óxido mixto sobre materiales de elevada área superficial evaluando dos métodos de incorporación. La actividad catalítica de los materiales se evaluó en la oxidación de Metil Fenil Sulfuro. 
Los materiales resultantes se caracterizaron mediante técnicas fisicoquímicas. El área superficial disminuyó respecto la matriz sin modificar como resultado de bloqueo de los poros y/o la ubicación de las partículas dentro de los poros de las zeolitas. Mediante difracción de rayos $\mathrm{X}$ se determinó que la muestra obtenida por mezcla mecánica presentó las señales de la zeolita pero con una disminución de la intensidad; la muestra "in-situ" no presentó estructura zeolítica ni cristalinidad. Los resultados de evaluación catalítica demostraron la importancia de la estructura y cristalinidad.

Palabras Claves: ÓXIDO MIXTO - ZEOLITA - OXIDACIÓN

\begin{abstract}
Mixed oxides present catalytic properties; however, they exhibit low surface area, which limits their use as catalysts. In order to increase their specific surface, materials were prepared incorporating a mixed oxide onto a material with elevated surface area evaluating two incorporation methods.

The catalytic activity of the materials was evaluated in the oxidation of Methyl Phenyl Sulfide.

The resulting materials were characterized by physicochemical techniques. The surface area decreased with respect to the unmodified matrix as a result of blocking the pores and/ or the location of the particles within the pores of the zeolites. By X-ray diffraction it was determined that the sample obtained by the mechanical mixture showed the zeolite signals but with a decrease in intensity; the "in-situ" sample did not present zeolitic structure or crystallinity. The results of catalytic evaluation demonstrated the importance of structure and crystallinity.
\end{abstract}

Keywords: MIXED OXIDE - ZEOLITE - OXIDATION

\title{
Introducción
}

Los óxidos mixtos con estructura tipo perovskitas poseen gran estabilidad térmica en un amplio rango de presiones parciales de oxígeno y presentan resistencia al envenenamiento catalítico (Pecchi et al., 2011), lo que ha centrado la atención en su uso como catalizadores para la oxidación catalítica de moléculas orgánicas.

El óxido mixto con estructura tipo perovskita, niobato de potasio (KNbO3) se ha reportado con aplicaciones interesantes en piezoelectricidad, piroelectricidad, electroóptica y comportamiento óptico no lineal (Nico et al., 2016). Además, también se han reportado interesantes propiedades catalíticas (Manzo et al., 2013; Wang y Li, 2012). En particular, se ha reportado en uso de niobato de potasio en procesos fotocatalíticos (Ding et al., 2008), oxidaciones de hollín (Pecchi et al., 2013) y reacciones de oxidación (Leal Marchena et al., 2016; Saux et al., 2016).

Sin embargo, las perovskitas puras preparadas a través de procedimientos convencionales exhiben un área superficial inferior a $30 \mathrm{~m} 2 / \mathrm{g}$ (Tejuca et al., 1989), lo que limita fuertemente la aplicación de estos materiales como catalizadores. Con el fin de aumentar su superficie 
específica, se han utilizado varios métodos de preparación para la síntesis de perovskitas, entre los que se destacan los métodos de sol-gel y citrato (Ciambelli et al., 2002), síntesis de combustión (Civera et al., 2005) y secado por pulverización (Sauvinet et al., 2002). Sin embargo, los resultados presentados demuestran que no es fácil mantener alta el área superficial y evitar procesos de sinterización simultáneamente. Una alternativa para superar esta limitación es soportar las perovskitas en matrices sólidas de elevada área superficial, tales como Al2O3 (Rosmaninho et al., 2010), SiO2 (Nguyen et al., 2002), tamices moleculares (Makshina et al., 2008) y zeolitas (Gu et al., 2015).

Las zeolitas son aluminosilicatos microporosos con estructura cristalina de poros uniformes y ordenados, con una elevada área superficial, estabilidad térmica, mecánica y química (Cundy y Cox, 2003).

La inmovilización de óxidos mixtos sobre las estructuras zeolíticas, materiales de gran superficie específica, permite obtener catalizadores heterogéneos fáciles de recuperar y reutilizar y con elevada área superficial.

La oxidación selectiva de sulfuros para obtener los correspondientes sulfóxidos y/o sulfonas es una reacción de transformación importante en la química orgánica. Los sulfóxidos y las sulfonas son moléculas bloques de construcción de alto potencial para compuestos orgánicos, como anti ulcerantes, antibacterianos y anti fúngicos, entre otros (Drabowski et al., 1994). Desde un punto de vista ambiental y económico, el uso de peróxido de hidrógeno como oxidante es particularmente útil debido a la seguridad en el almacenamiento y la operación, el bajo costo, el contenido de oxígeno altamente efectivo y el hecho de que sólo genera agua como producto secundario. Sin un catalizador metálico, la oxidación de sulfuro con peróxido de hidrógeno requiere altas temperaturas y/o largos tiempos de reacción (Jereb, 2012). Por lo tanto, la optimización de catalizadores y/o los sistemas de reacción permite obtener una oxidación altamente selectiva del sulfuro en condiciones de reacción suaves.

Basándonos en estudios previos de nuestro laboratorio (Leal Marchena et al., 2013) y a los fines de acotar las variables de análisis, se seleccionó incorporar un 30\%p/p del óxido mixto, planteando como objetivo la obtención de materiales catalíticos a partir de la incorporación niobato de potasio sobre zeolita BETA evaluando dos métodos de incorporación. Los materiales resultantes se evaluarán en la oxidación de Metil Fenil Sulfuro en condiciones suaves de reacción.

\section{Metodología}

\section{Síntesis de catalizadores}

La zeolita BETA se sintetizó mediante el método de cristalización hidrotérmica utilizando hidróxido de tetraetilamonio como agente de dirección (Valencia et al., 1995). La forma amonio de zeolita se preparó mediante intercambio iónico de la forma sódica de la zeolita preparada con una solución de cloruro de amonio $1 \mathrm{M}$ a $80^{\circ} \mathrm{C}$ durante $40 \mathrm{~h}$.

El gel del niobato de potasio (KNbO3) se obtuvo a partir del método de citrato (Wang et al., 2007) empleando acetato de potasio, peróxido de hidrógeno (H2O2) y ácido cítrico como materiales de partida. Una solución acuosa del acetato alcalino se mezcló a temperatura ambiente con una cantidad estequiométrica de $\mathrm{NbCl5}$ previamente disuelta en $\mathrm{H} 2 \mathrm{O} 2$ bajo agitación constante. Se añadió ácido cítrico con agitación constante en una relación molar de 3 ácido cítrico/catión metálico, obteniéndose un gel.

Se evaluaron dos métodos de incorporación del KNbO3 para obtener un 30\%p/p sobre la 
zeolita:

1. In situ (KNbBETAis): se goteó el gel de KNbO3 lentamente sobre la zeolita, la cual se encontraba humedecida con etanol para evitar el intercambio iónico a temperatura ambiente. La mezcla se agitó durante $5 \mathrm{~h} \mathrm{a} 60^{\circ} \mathrm{C}$ y luego en horno a igual temperatura durante $24 \mathrm{~h}$. El material final se calcinó a $600^{\circ} \mathrm{C}$ durante $12 \mathrm{~h}$.

2. Mezcla mecánica (KNbBETAmm): el segundo método consistió en una mezcla física del gel $\mathrm{KNbO} 3$ secado a $60^{\circ} \mathrm{C}$ durante $24 \mathrm{~h}$ y la zeolita. El material final se calcinó en horno a $600^{\circ} \mathrm{C}$ durante $12 \mathrm{~h}$.

\section{Caracterización}

El área superficial específica (SBET) se determinó a partir de las mediciones de desorción de nitrógeno a $77 \mathrm{~K}$ utilizando equipos TriStar II 3020. Previamente, las muestras se desgasificaron a $300^{\circ} \mathrm{C}$ durante $2 \mathrm{~h}$. Los espectros de difracción de rayos X (XRD) se obtuvieron utilizando un difractómetro D4 Endeavor Bruker AXS equipado con una radiación CuKa filtrada con níquel $(\lambda=1.5418 \AA$ ). Las fases se identificaron comparando los patrones de difracción con los archivos de difracción de referencia en el software EVA. Los espectros de Infrarrojo con Transformada de Fourier (FTIR) se determinaron utilizando equipo Nicolet Nexus.

\section{Evaluación catalítica}

La oxidación catalítica de Metil Fenil Sulfuro (MFS, Aldrich) empleando H2O2 (30\%, Cicarelli) como agente oxidante se llevó a cabo en un reactor de vidrio pirex sumergido en un baño termostatizado equipado con un condensador a reflujo y con agitación magnética vigorosa. Se empleó como solvente acetonitrilo (99,5\% Cicarelli) y una temperatura de $40^{\circ} \mathrm{C}$. Los productos orgánicos fueron extraídos a diferentes tiempos de reacción durante $4 \mathrm{~h}$ de reacción, se analizaron y cuantificaron por cromatografía en fase gaseosa (CG) empleando un cromatógrafo Perkin Elmer 500 equipado con columna capilar (ZB-1) y detector FID, y por espectrometría de masas empleando un equipo GC-Masas (Shimadzu QP 5050 GC-17 A).

\section{Resultados}

\begin{tabular}{|l|l|}
\hline & $\mathrm{S}_{\mathrm{BET}}\left(\mathrm{m}^{2} \mathrm{~g}^{-1}\right)$ \\
\hline $\mathrm{KNbO}_{3}$ & 10,5 \\
\hline BETA & 485,3 \\
\hline KNbBETA $_{\mathrm{mm}}$ & 263,7 \\
\hline KNbBETA $_{\text {is }}$ & 228,6 \\
\hline
\end{tabular}

Tabla 1. Área superficial específica de KNbO3, BETA y las muestras obtenidas. 


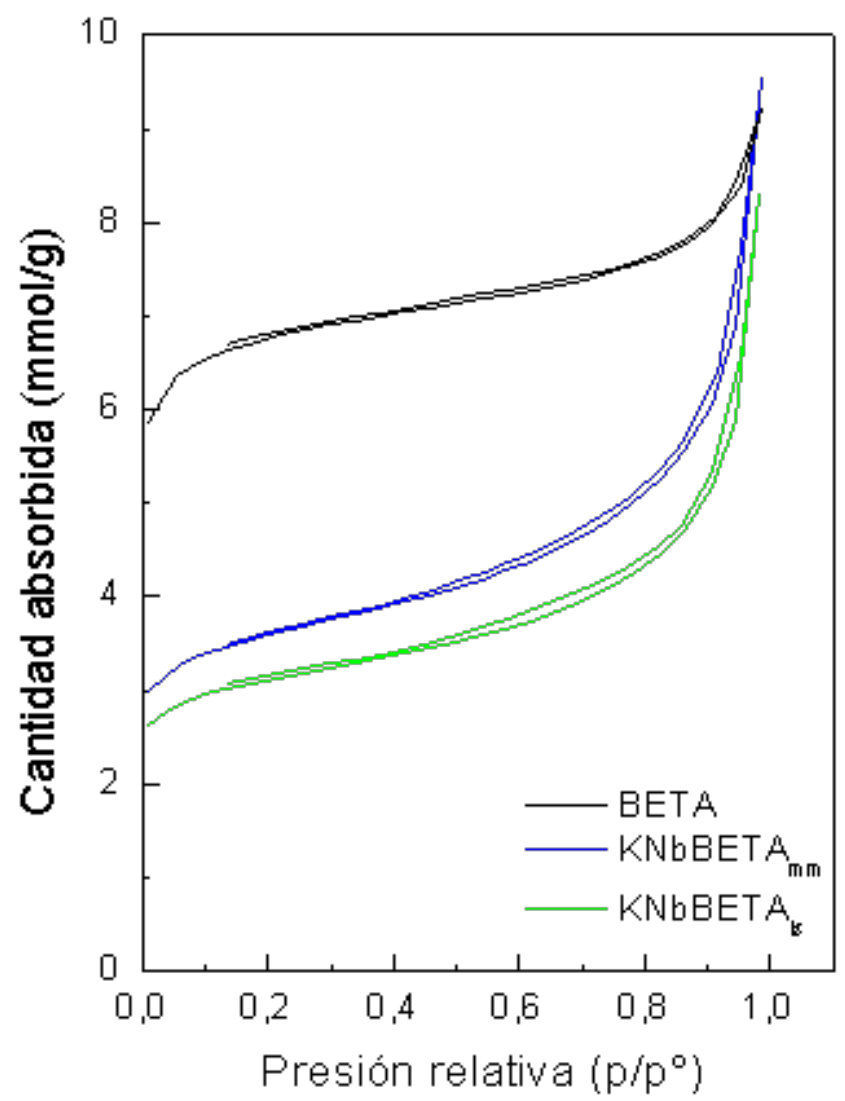

Figura 1. Isotermas de absorción-desorción de N2 de BETA, KNbBETAmm y KNbBETAis.

De acuerdo con la clasificación IUPAC, las isotermas de absorción-desorción N2 de la zeolita BETA se puede clasificar como Tipo I, característica de los sólidos microporosos. El SBET de los materiales, la zeolita BETA y el $\mathrm{KNbO}$ determinada a partir de las isotermas de adsorción-desorción de N2 utilizando el método Brunauer-Emmett-Teller (BET) se presentan en la Tabla 1. Las isotermas de las muestras modificadas exhiben características similares a las de la zeolita respectiva. Los valores de SBET disminuyen cuando se incorporó el niobato independientemente del método utilizado. La progresiva disminución de SBET podría deberse a la obstrucción de poros de zeolita por parte de los óxidos o bien por la ubicación de las partículas dentro del espacio poroso de las zeolitas (lo que limita la accesibilidad de las moléculas de gas). Como lo indica la menor cantidad de gas adsorbido (Figura 1), todos los óxidos metálicos podrían penetrar en los poros de la zeolita (Chagas et al., 2012). 


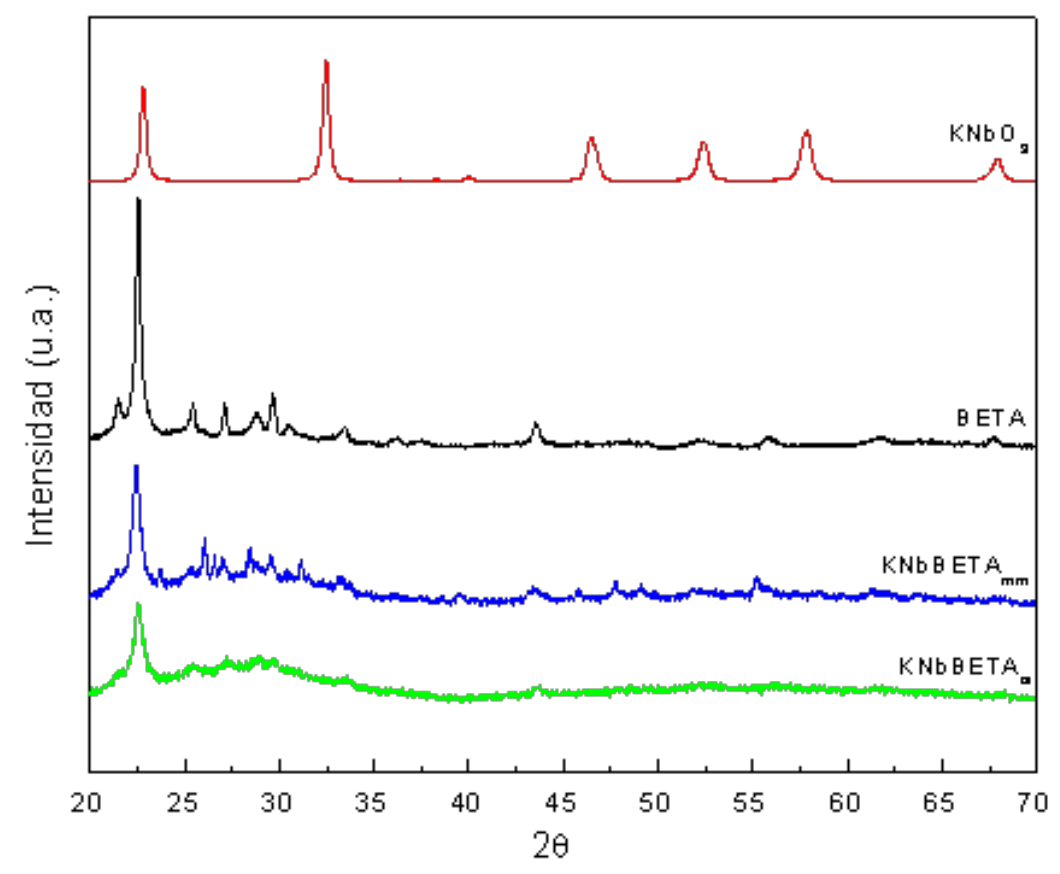

Figura 2. Patrones de XRD de KNbO3, BETA, KNbBETAmm y KNbBETAis.

En la Figura 2 se presentan los patrones de difracción de XRD del niobato de potasio, la zeolita BETA y las muestras obtenidas con ambos métodos. El patrón de difracción del KNbO3 es característico de la estructura perovskita cristalina ortorrómbica (JCPDS 32822) (Russo et al., 2008). La zeolita BETA presenta las señales características a $2 \Theta=21$ y $22^{\circ}$ con estructura cristalina (Renzini et al., 2009) (JCPDS 48-0074). En el patrón de la muestra KNbBETAmm están presentes las señales características de la zeolita pero se observa una disminución de la intensidad. La ausencia de las señales del óxido puede deberse a la incorporación del óxido en los poros, tal como se determinó por BET o a que los óxidos se encuentran distribuidos en la superficie y no se detectan por esta técnica dado que las señales de la zeolita son más intensas produciéndose un efecto de dilución. La muestra KNbBETAis no posee estructura zeolítica ni cristalinidad. La pérdida de la estructura podría deberse a la acidez de los precursores del óxido mixto que generan la destrucción de la zeolita junto con los tratamientos térmicos a los que se somete. 


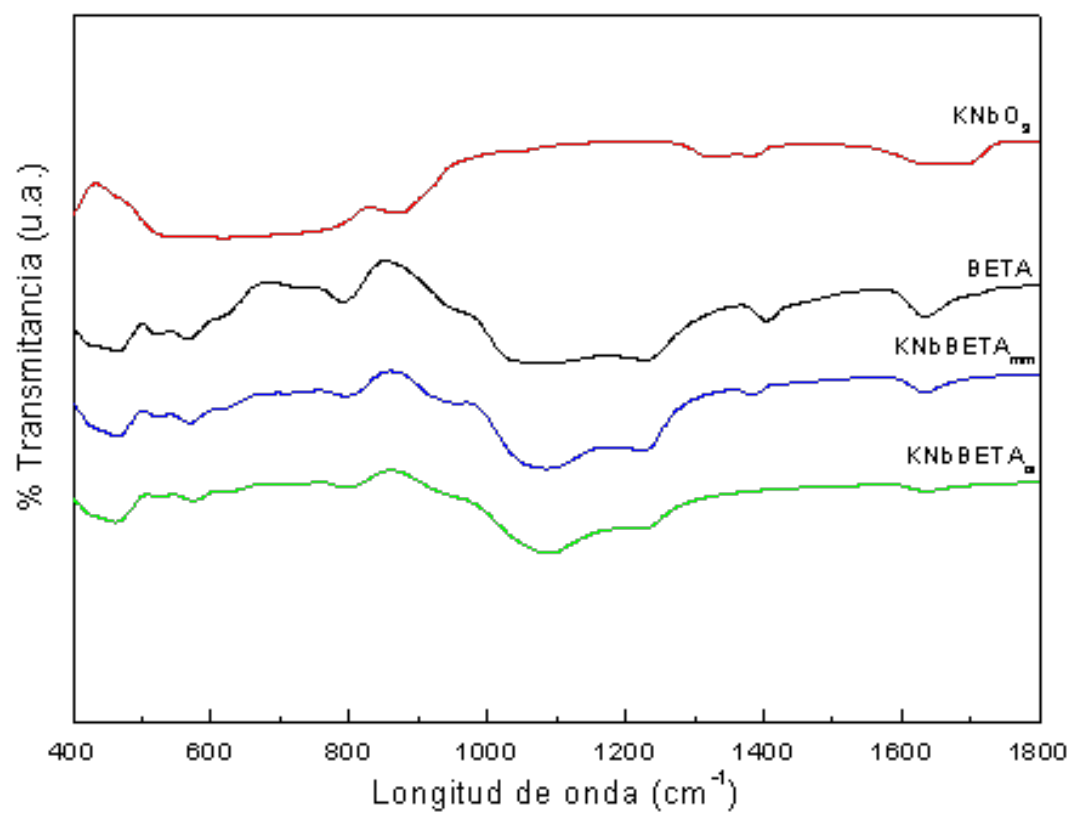

Figura 3. Espectro de FTIR de KNbO3, BETA, KNbBETAmm y KNbBETAis.

El espectro de FTIR de KNbO3 (Figura 3) muestra una señal a $600 \mathrm{~cm}-1$, la cual es atribuida a la vibración de los octaedros de NbO6. La señal a $850 \mathrm{~cm}-1$ es asignada al estiramiento asimétrico del enlace $\mathrm{Nb}-\mathrm{O}$ de las estructuras octaédricas de $\mathrm{NbO6}$, lo que indica la presencia de la estructura cristalina de perovskita, tal como se corroboró mediante XRD; y la banda a $1700 \mathrm{~cm}-1$ corresponde a la vibración de los grupos CO3-2 (Belmokhtar et al., 2015). El espectro de la zeolita BETA presenta las cuatro señales características de la estructura zeolítica a $550,620,800$ y $1230 \mathrm{~cm}-1$. El enlace a $550 \mathrm{~cm}-1$ se asigna a las vibraciones T-O-T ( $\mathrm{T}=\mathrm{Si}$ o $\mathrm{Al}$ ) de los anillos de 5 y 6 miembros en la estructura de la zeolita, lo que indica cristalinidad de zeolita. Las otras tres señales se originan debido a las vibraciones del estiramiento simétrico O-T-O, al estiramiento asimétrico interno O-T-O y al estiramiento asimétrico externo O-T-O, respectivamente. El pico a $1640 \mathrm{~cm}-1$ se atribuye a la vibración de los enlaces O-H del agua adsorbida (Ebrahiminejad y Karimzadeh, 2019). En los patrones de las muestras sintetizadas no se detectan los picos del niobato, similar a lo observado mediante XRD; se observa una correlación entre la intensidad de las señales y el nivel de cristalinidad para la parte de zeolita de las muestras sintetizadas (Zhang et al., 2014).

Figura 4. Conversión de MFS en función del tiempo de reacción. Condiciones de reacción: MFS (2 mmol), H2O2 (4 mmol), catalizador $(20 \mathrm{mg})$, acetonitrilo (10 $\mathrm{mL})$, temperatura: $40{ }^{\circ} \mathrm{C}$.

La inmovilización de óxido mixto sobre zeolitas es una forma común de superar el inconveniente de la baja área superficial que posee la perovskita (Zhu et al., 2015); a los fines de evaluar los dos métodos de incorporación se determinó la actividad catalítica de los mismos en la oxidación de MFS (Figura 4).

Cuando se empleó zeolita BETA como catalizador para la oxidación, la conversión de 
sulfuro aumenta gradualmente con el tiempo de reacción alcanzando una conversión final de 29,5 mol\% luego de 4 h de reacción. En el caso del KNbO3, se obtuvo una conversión final de 40,5 mol\%, mientras que al emplear la contraparte soportada mediante mezcla mecánica, se alcanzó un 58,4 mol\% de conversión final. Para la muestra obtenida a partir de la síntesis in-situ, la conversión alcanzada luego de 4 h de reacción fue de sólo 18,6 mol\%. KNbBETAmm resultó el catalizador más activo de todas las muestras evaluadas para esta reacción, siendo el sulfóxido y la sulfona correspondientes los únicos productos obtenidos en todos los casos. Incluso a pesar que el óxido mixto (KNbO3) y la matriz de zeolita (BETA) resultaron activas para la oxidación, la muestra soportada mediante mezcla mecánica resultó más activa que ellas. Especialmente cuando se considera que en todos los casos se emplearon $20 \mathrm{mg}$ de cada catalizador y sólo contiene $30 \%$ p/p del óxido mixto.

Los resultados de actividad catalítica de las perovskitas dependen en gran medida de la estructura y sus defectos, lo que beneficia las vacancias de oxígeno y la movilidad de oxígeno de la red. A menor cristalinidad, mayor movilidad de oxígeno, el cual resulta fundamental para las reacciones de oxidación (Alifanti et al., 2003). Sin embargo, para el caso de la muestra obtenida mediante síntesis in-situ no se logró obtener la estructura perovskita ni se mantuvo la estructura zeolítica resultando en un material amorfo.

La dispersión del óxido mixto mediante mezcla mecánica sobre la matriz zeolítica aumentó el área de exposición del KNbO3 favoreciendo el contacto de los sitios activo-sustrato oxidante.

\section{Conclusiones}

Se evaluaron dos métodos de incorporación de óxido mixto con estructura tipo perovskita sobre materiales con elevada área superficial. Tanto los materiales sin soportar como los materiales soportado se caracterizaron y se evaluaron sus propiedades catalíticas en la oxidación selectiva de Metil Fenil Sulfuro en condiciones suaves de reacción.

Los resultados de la caracterización mostraron que el método de citrato resulta adecuado para obtener la estructura tipo perovskita del $\mathrm{KNbO}$, pero el procedimiento para soportar la perovskita sobre la zeolita mediante la síntesis in-situ generó cambios estructurales en la estructura zeolítica resultando en una estructura amorfa causado posiblemente por la acidez de los precursores de síntesis y sin actividad catalítica considerable. El método de mezcla mecánica resultó adecuado, evidenciado en los resultados de actividad catalítica. La actividad presentada se puede atribuir a su mayor área expuesta de perovskita disponible y a una ligera disminución de la cristalinidad que permite una mayor movilidad del oxígeno de la red, una propiedad esencial en las reacciones de oxidación.

\section{Agradecimientos}

Al Ministerio de Ciencia y Tecnología de la Provincia de Córdoba (GRFT 2017 Resol. N ${ }^{\circ}$ 109/17), a la Universidad Tecnológica Nacional (PID 4356 y UTI3864TC) y al Consejo Nacional de Investigaciones Científicas y Técnicas. 


\section{Referencias}

Alifanti, M., Kirchnerova, J. y Delmon, B. (2003) "Effect of substitution by cerium on the activity of LaMnO3 perovskite in methane combustion”, Applied Catalysis A: General, 245, 231-244.

Belmokhtar, N., Brahimi, R., Nedjar, R. y Trari, M. (2015) "Preparation and physical properties of the layered niobate $\mathrm{Cu} 0.5 \mathrm{Nb308}$ : Application to photocatalytic hydrogen evolution", Materials Science in Semiconductor Processing, 39, 433-440.

Chagas, C.A., Souza Toniolo, F., Newton, R., Magalhães, S.H. y Schmal, M. (2012) "Aluminasupported LaCoO3 perovskite for selective CO oxidation (SELOX)", International Journal of Hydrogen Energy, 37, 5022-5031.

Ciambelli, P., Cimino, S., Lasorella, G., Lisi, L., De Rossi, S., Faticanti, M., Minelli, G. y Porta, P. (2002) "CO oxidation and methane combustion on LaAl1-xFexO3 perovskite solid solutions”, Applied Catalysis B: Environmental, 37, 231-241.

Civera, A., Negro, G., Specchia, S. y Saracco, G. (2005) "Optimal compositional and structural design of a LaMnO3/ZrO2/Pd-based catalyst for methane combustion", Catalysis Today, 100, 275-281.

Cundy, C.S. y Cox, P.A. (2003) "The Hydrothermal Synthesis of Zeolites: History and Development from the Earliest Days to the Present Time", Chemical Reviews, 103, 663-701.

Ding, Q., Yuan, Y., Xiong, X., Li, R., Huang, H., Li, Z., Yu, T., Zou, Z. y Yang, S. (2008) "Enhanced photocatalytic water splitting properties of KNbO3 nanowires synthesized through hydrothermal method", Journal of Physical Chemistry C, 112, 18846-18848.

Drabowski, J., Kielbasinski, P., Mikolajcyk, M. (1994) Synthesis of Sulfoxides. New York: John Wiley and Sons.

Ebrahiminejad, M. y Karimzadeh, R. (2019) "Hydrocracking and hydrodesulfurization of diesel over zeolite beta-containing NiMo supported on activated red mud", Advanced Powder Technology, 30, 1450-1461.

Gu, X., Lu H., Kan, C. y Yao J. (2015) “One-pot hydrothermal synthesis of zeolite/sodium tantalate composite and its photodegradation of methyl orange”, Materials Research Bulletin, 68, 185-188.

Jereb, M. (2012) "Highly atom-economic, catalyst- and solvent-free oxidation of sulfides into sulfones using 30\% aqueous H2O2”, Green Chemistry, 14, 3047-3052.

Leal Marchena, C., Frenzel, R.A., Gomez, S., Pierella, L.B. y Pizzio, L.R. (2013)

"Tungstophosphoric acid immobilized on ammonium Y and ZSM5 zeolites: Synthesis, characterization and catalytic evaluation”, Applied Catalysis B: Environmental, 130- 131, 187- 196.

Leal Marchena, C., Saux, C., Dinamarca, R., Pecchi, G. y Pierella, L. (2016) "Alkaline niobates ANbO3 ( $\mathrm{A}=\mathrm{Li}, \mathrm{Na}, \mathrm{K})$ as heterogeneous catalysts for dipropyl sulfide oxidation", RSC Advances, 6, 102015-102022.

Makshina, E.V., Nesterenko, N.S., Siffert, S., Zhilinskaya, E.A., Aboukais, A. y Romanovsky, B.V. (2008) "Methanol oxidation on LaCo mixed oxide supported onto MCM-41 molecular sieve”, Catalysis Today, 131, 427-430. 
Manzo, M., Laurell, F., Pasiskevicius, V. y Gallo, K. (2013). Nano-Optics for Enhancing Light-Matter Interactions on a Molecular Scale. Netherlands: Springer, 421-422.

Nguyen, S.V., Szabo, V., Trong On, D.y Kaliaguine S. (2002) “Mesoporous silica supported LaCoO3 perovskites as catalysts for methane oxidation", Microporous and Mesoporous Materials, 54, 51-61.

Nico, C., Monteiro, T. y Graça, M.P.F. (2016) "Niobium oxides and niobates physical properties: Review and prospects”, Progress in Materials Science, 80, 1-37.

Pecchi, G., Cabrera, B., Delgado, E., Gordon, A. y Jimenez, R. (2013) "Catalytic oxidation of soot over alkaline niobates", Journal of Alloys and Compounds, 551, 255-261.

Pecchi, G., Campos, C. y Peña, O. (2011) "Catalytic performance in methane combustion of rare-earth perovskites RECo0.50Mn0.5003 (RE: La, Er, Y)”, Catalysis Today, 172, 111-117

Renzini, M.S., Sedrán, U. y Pierella, L.B. (2009) "H-ZSM-11 and Zn-ZSM-11 zeolites and their applications in the catalytic transformation of LDPE", Journal of Analytical and Applied Pyrolysis, 86, 215-220.

Rosmaninho, M.G., Tristao, J.C., Moura, F.C.C., Lago, R.M., Araujo, M.H. y Fierro, J.L. (2010) "Structural and surface analysis of unsupported and alumina-supported La(Mn, Fe, Mo)O3 perovskite oxides", Analytical and Bioanalytical Chemistry, 396, 2785-2795.

Russo, N., Furfori, S., Fino, D., Saracco, G. y Specchia, V. (2008) "Lanthanum cobaltite catalysts for diesel soot combustion”, Applied Catalysis B: Environmental, 83, 85-95.

Sauvinet, A.L., Fouletier, J., Gaillard, F. y Primet, M. (2002) "Surface Properties and Physicochemical Characterizations of a New Type of Anode Material, La1-xSrxCr1yRuyO3- $\delta$, for a Solid Oxide Fuel Cell under Methane at Intermediate Temperature", Journal of Catalysis, 209, 25-34.

Saux, C., Leal Marchena, C., Pecchi, G. y Pierella, L. (2016) "Active potassium niobates and titanoniobates as catalysts for organic sulfide remediation", Catalysis Communications, 76, 58-61.

Tejuca, L., Fierro, J.L., Tascón, J.M. (1989) "Structure and reactivity of perovskite-type oxides”, Advances in Catalysis, 36, 237-328.

Valencia, S., Camblor, M.A., Corma, A. y Perez Pariente, J., “Síntesis de zeolita Beta”, Pat. Num. 2124142 - Patente Española - España Agosto 1 (1995).

Wang, K. y Li, J.F. (2012) “(K, Na)NbO3-based lead-free piezoceramics: Phase transition, sintering and property enhancement”, Journal of Advanced Ceramics, 1, 24-37.

Wang, L.H., Yuan, D.R., Duan, X.L., Wang, X.Q. y Yu, F.P. (2007) "Synthesis and characterization of fine lithium niobate powders by sol- gel method”, Crystal Research \& Technology, 42, 321-324.

Zhang, Q., Ming, W., Ma, J., Zhang, J., Wang, P. y Li, R. (2014) “De novo assembly of a mesoporous beta zeolite with intracrystalline channels and its catalytic performance for biodiesel production”, Journal of Materials Chemistry A, 2, 8712-8718.

Zhu, H., Zhang, P. y Dai, S. (2015) "Recent Advances of Lanthanum-Based Perovskite Oxides for Catalysis”, ACS Catalysis, 5, 6370-6385. 